\title{
Treating Diet-Induced Obesity: A New Role for Vagal Afferents?
}

\author{
Edward A. Fox
}

Received: 27 February 2012/ Accepted: 1 March 2012/Published online: 22 March 2012

(C) Springer Science+Business Media, LLC 2012

As of the 2007-2008 National Health and Nutrition Examination Survey, $34 \%$ of adults-or over 72 million people—were obese, having a BMI $>30$. With childhood obesity catching up, this epidemic will only continue to grow for the foreseeable future. The current "obesogenic" environment characteristic of developed countries is often cited as the major cause of the obesity epidemic. Nevertheless, the observation that a large percentage of the population has not become obese could imply the contribution of a gene-environment interaction. For instance, the differential response to the obesogenic environment suggests one's inherited physiological makeup (which itself is modulated by environment) either resists or favors obesity.

Being obese might not be so unfortunate if weight gain and increased fat mass were the only consequences. Nonetheless, obesity is associated with numerous consequences such as diabetes, cardiovascular disease, and cancer [1], which in turn are related to proportionately large visceral abdominal fat stores [2]. Regrettably, no satisfactory non-invasive treatment for obesity has been developed. As a consequence, increasing numbers of expensive and risky bariatric surgical procedures, the only effective current therapies, are performed. Gastric bypass, though reasonably effective at reducing body weight, has been useful for treating type 2 diabetes [3]. The mechanism underlying these effects is not fully understood, but appears to involve decreases in consumption of high calorie foods, reductions of meal size and nutrient absorption, changes of anorexigenic and orexigenic hormone levels, increased

\section{E. A. Fox $(\square)$}

Behavioral Neurogenetics Laboratory, Ingestive Behavior Research Center, Department of Psychological Sciences, Purdue University, 703 Third Street, West Lafayette, IN 47907, USA e-mail: au_gc@psych.purdue.edu energy expenditure, and altered vagal gastrointestinal (GI) innervation [4]. The key to the success of bypass in reducing body weight may thus be due to its effects on multiple organ systems.

Before bariatric procedures became popular, truncal vagotomy, involving division of the right and left vagal trunks below the diaphragm was employed to treat morbid obesity [5]. Nonetheless, the interruption of vagal premotor axons as part of the vagotomy procedure caused gastric stasis and "dumping" of food from the stomach to the intestine, causing unpleasant sensations, including nausea. One way to overcome the adverse side effects of vagotomy and retain some of the benefits was to reduce or avoid damage to the vagal pre-motor axons. Indeed, selective sensory vagotomy may retain some of the benefits as it reduces food intake, body weight, and fat accumulation in rodent obesity models [6, 7]. Moreover, sensory vagotomy reduced the excess abdominal visceral fat found in aging rats [7]. Stearns et al. [8] took this approach further by distinguishing the effects of sensory vagotomy on different fat depots in rats subjected to diet-induced obesity (DIO). Sensory vagotomy was achieved by applying capsaicin to the abdominal vagal branches, and comparing the effect of this treatment on DIO in rats fed a high-energy "Western diet" for 11 months with the effects of truncal vagotomy and sham surgery. Interestingly, although sensory vagotomy non-significantly reduced body weight, it also modestly, but significantly and selectively, reduced visceral abdominal fat by $18 \%$ compared with controls. This is an important finding given the aforementioned association of central obesity with several causes of morbidity and mortality and since small changes in the size of the visceral fat depot can exert sizeable influences on overall mortality risk [9]. Stearns et al. [8] further suggested that sensory vagotomy could provide a valuable 
adjunct to bariatric surgery by enhancing its ability to reduce body fat, and in particular visceral fat. Consistent with this possibility, truncal vagotomy has been used successfully as an adjunct to gastroplasty to augment weight loss [10].

Another important implication of the findings of Stearns et al. [8] is that vagal GI afferents may contribute to DIO. However, this interpretation is counter to traditional views of vagal GI sensory function in two ways. First, the principal functions of vagal afferents are thought to be inhibition of food intake [11] and regulation of vago-vagal digestive reflexes [12] — not facilitation of food intake or weight gain. Second, the influences of vagal afferents on food intake are thought to act on a short-term, or meal-bymeal basis_-not to accrue over the long-term [11].

As noted by Stearns et al. [8], intestinal mucosal afferent nerves may influence the formation of visceral adipose tissue [17], and therefore, if these nerves include vagal afferents, this would be a direct pathway they could utilize to promote abdominal obesity. Another route by which vagal afferents could facilitate food intake that might lead to weight gain and fat accumulation involves alterations to their control of vago-vagal GI reflexes. For example, accommodation is activated by food-induced stretch of the esophagus and the forestomach [13]. It relaxes the stomach wall, which is contracted between meals. This enables the stomach to accept a meal without significantly increasing intragastric pressure. Exaggeration of this relaxation response could permit consumption of larger-than-normal meals before intragastric pressure is increased sufficiently to activate vagal satiation signals, or to propel food into the intestine, which also activates these signals. Consistent with this possibility, loss of vagal afferents that innervate the forestomach in mice with mutations of either the $c$-Kit tyrosine kinase receptor, or its ligand steel factor reduced meal size, which implies that reduced vagal innervation impaired accommodation and thus reduced the stomach volume available to accept food (e.g. [14]). Also, increased meal size associated with knockout $(\mathrm{KO})$ of brain-derived neurotrophic factor (BDNF) in GI smooth muscle was associated with increased activation of gastric vagal premotor neurons, an effect consistent with augmentation of accommodation or gastric motility [15, 16]. However, these suspected changes in vago-vagal reflexes in steel, $W^{v}$, and BDNF mutants need to be verified.

Only a handful of studies have provided supporting evidence for long-term effects of vagal GI afferents that might include accumulation of the visceral fat component of body weight. These studies involved reductions in central and peripheral CCK receptors that resulted in obesity [18], intracerebroventricular leptin injection paired with subthreshold peripheral CCK infusion that reduced body weight [19]. These peripheral CCK effects presumably involved altered vagal afferent signaling. Additionally, consumption of a high-energy diet by neurotrophin- $4 \mathrm{KO}$ mice, which lack most of the vagal afferents that innervate the smooth muscle of the small intestine, revealed an increased hyperphagia compared to controls [16, 20]. Interestingly, the vagal manipulations in these studies have typically occurred in conjunction with alterations of the CNS feeding regulatory circuit, suggesting these alterations are needed to reveal a long-term vagal afferent role.

A possibility to consider is that the long-term effects associated with the loss of vagal afferents in neurotrophin4 mutants and in the DIO rats reported by Stearns et al. [8] involved changes to the CNS feeding regulatory circuit. High-energy diets can reduce the levels of anorexigens in CNS nuclei that regulate feeding and body weight [21]; in some instances these reductions contribute to hyperphagia and obesity [22]. Thus, if the high-energy diets fed to the neurotrophin-4 mutants or the DIO rats of Stearns et al. [8] reduced anorexigen levels in key CNS nuclei, then these reductions, combined with the effects of the missing vagal GI afferents, may have produced long-term effects on food intake and abdominal fat. Finally, as noted by Stearns et al. [8], clinical studies using chronic electrical stimulation of the cervical vagus which induced weight loss in depressed obese patients is consistent with a long-term role for vagal afferents in body weight regulation.

To better understand the contributions of changes in feeding behavior and energy expenditure to the effects of vagotomy on abdominal fat, it would be valuable to characterize them early in the dynamic phase of weight gain. Obesity can independently affect food intake and energy expenditure, confounding the effects of vagotomy. Therefore, measuring food intake and energy expenditure prior to development of obesity will help establish whether changes in these variables are a cause or a consequence of obesity. Another valuable addition would be to examine the effect of vagotomy on groups receiving high-energy diet or a normal energy diet balanced for key components. Groups that receive no treatment, sham surgery or vagotomy and are fed normal energy-diet provide baselines that permit one to distinguish, for example, the actual degree of obesity in each group, rather than just the relative degree. Also, the proportions of the obesity and excess visceral fat due to aging [7] versus diet [8] could be distinguished, both with and without vagotomy. Further, since capsaicin only partially reduces the number of vagal GI afferents, it might be useful to assess residual vagal function, e.g. by examining CCK suppression of meal size before exposure to a highenergy diet. In addition to confirming the success of the vagotomy, these types of experiments could help identify the vagal sensory pathways that contribute to DIO. Finally, when lengthy postvagotomy survival times are involved, vagal afferents regenerate [23]; therefore, this should be 
examined because it could actually mask some of the beneficial effects of vagotomy on DIO.

Studies that build on the findings of Stearns et al. [8] by considering these and possibly additional useful variations of the experimental design will bring us closer to knowing whether vagal GI afferents contribute to long-term regulation of food intake and abdominal fat and if so how they do this. Such progress will provide novel targets for gaining control over the excessive overeating and weight gain that has become so pervasive.

Acknowledgments This research is supported by Grant NS046716.

Conflict of interest None.

\section{References}

1. Hjartaker A, Langseth $\mathrm{H}$, Weiderpass E. Obesity and diabetes epidemics: cancer repercussions. Adv Exp Med Biol. 2008;630:72-93.

2. Kishida K, Funahashi T, Matsuzawa Y, Shimomura I. Visceral adiposity as a target for the management of the metabolic syndrome. Ann Med. 2011;25:25.

3. Serrot FJ, Dorman RB, Miller CJ, et al. Comparative effectiveness of bariatric surgery and nonsurgical therapy in adults with type 2 diabetes mellitus and body mass index $<35 \mathrm{~kg} / \mathrm{m}^{2}$. Surgery. 2011;150:684-691.

4. Ionut V, Bergman RN. Mechanisms responsible for excess weight loss after bariatric surgery. J Diabetes Sci Technol. 2011; 5:1263-1282.

5. Kral JG, Gortz L. Truncal vagotomy in morbid obesity. Int J Obes. 1981;5:431-435.

6. Ferrari B, Arnold M, Carr RD, et al. Subdiaphragmatic vagal deafferentation affects body weight gain and glucose metabolism in obese male Zucker (fa/fa) rats. Am J Physiol Regul Integr Comp Physiol. 2005;289:R1027-R1034.

7. Melnyk A, Himms-Hagen J. Resistance to aging-associated obesity in capsaicin-desensitized rats 1 year after treatment. Obes Res. 1995;3:337-344.

8. Stearns AT, Balakrishnan A, Radmanesh A, Ashley SW, Rhoads DB, Tavakkolizadeh A. Relative contributions of afferent vagal fibers to resistance to diet-induced obesity. Dig Dis Sci. (Epub ahead of print). doi:10.1007/s10620-011-1968-4.

9. Kuk JL, Katzmarzyk PT, Nichaman MZ, Church TS, Blair SN, Ross R. Visceral fat is an independent predictor of all-cause mortality in men. Obesity (Silver Spring). 2006;14:336-341.
10. Kral JG, Gortz L, Hermansson G, Wallin GS. Gastroplasty for obesity: long-term weight loss improved by vagotomy. World $J$ Surg. 1993;17:75-78, discussion 79 .

11. Berthoud HR. The vagus nerve, food intake and obesity. Regul Pept. 2008;149:15-25.

12. Kitamura A, Torii K, Uneyama H, Niijima A. Role played by afferent signals from olfactory, gustatory and gastrointestinal sensors in regulation of autonomic nerve activity. Biol Pharm Bull. 2010;33:1778-1782.

13. Takahashi T, Owyang C. Characterization of vagal pathways mediating gastric accommodation reflex in rats. $J$ Physiol. 1997;504:479-488.

14. Fox EA, Phillips RJ, Byerly MS, Baronowsky EA, Chi MM, Powley TL. Selective loss of vagal intramuscular mechanoreceptors in mice mutant for steel factor, the c-kit receptor ligand. Anat Embryol. 2002;205:325-342.

15. Biddinger JE, Fox EA. Meal pattern and microstructure changes underlying hyperphagia and obesity in mice with smooth musclespecific brain-derived neurotrophic factor knockout [abstract]. Soc Neurosci Abstr. 2010. Abstract no. 391.10.

16. Fox EA, Biddinger JE, Jones KR, Worman A, McAdams J. Smooth muscle-specific knockout of brain-derived neurotrophic factor results in hyperphagia and obesity [abstract]. Soc Neurosci Abstr. 2010. Abstract no. 391.7.

17. Leung FW, Go VL, Scremin OU, et al. Pilot studies to demonstrate that intestinal mucosal afferent nerves are functionally linked to visceral adipose tissue. Dig Dis Sci. 2007;52:2695-2702.

18. Bi S, Scott KA, Kopin AS, Moran TH. Differential roles for cholecystokinin a receptors in energy balance in rats and mice. Endocrinology. 2004;145:3873-3880.

19. Matson CA, Reid DF, Cannon TA, Ritter RC. Cholecystokinin and leptin act synergistically to reduce body weight. Am J Physiol Regul Integr Comp Physiol. 2000;278:R882-R890.

20. Byerly MS, Fox EA. High-fat hyperphagia in neurotrophin-4 deficient mice reveals potential role of vagal intestinal sensory innervation in long-term controls of food intake. Neurosci Lett. 2006;400:240-245.

21. Yu Y, Wang Q, Huang XF. Energy-restricted pair-feeding normalizes low levels of brain-derived neurotrophic factor/tyrosine kinase B mRNA expression in the hippocampus, but not ventromedial hypothalamic nucleus, in diet-induced obese mice. Neuroscience. 2009;160:295-306.

22. Unger TJ, Calderon GA, Bradley LC, Sena-Esteves M, Rios M. Selective deletion of Bdnf in the ventromedial and dorsomedial hypothalamus of adult mice results in hyperphagic behavior and obesity. J Neurosci. 2007;27:14265-14274.

23. Phillips RJ, Baronowsky EA, Powley TL. Regenerating vagal afferents reinnervate gastrointestinal tract smooth muscle of the rat. J Comp Neurol. 2000;421:325-346. 